\title{
A multidisciplinary quality improvement effort to reduce bronchopulmonary dysplasia incidence
}

\author{
Roopali Bapat $^{1} \cdot$ Leif Nelin ${ }^{1,2} \cdot$ Edward Shepherd ${ }^{1} \cdot$ Greg Ryshen $^{3} \cdot$ Amee Elgin $^{3} \cdot$ Thomas Bartman $^{1}$
}

Received: 23 July 2019 / Revised: 3 December 2019 / Accepted: 18 December 2019 / Published online: 6 January 2020

(C) Springer Nature America, Inc. 2020

\begin{abstract}
Background Bronchopulmonary Dysplasia (BPD) is the most common prematurity complication. Although several practices have been proposed for BPD prevention, none of these in isolation prevent BPD.

Methods Our initiative focused on two key drivers: oxygen management and noninvasive ventilation strategies. We created best practice guidelines and followed outcome measures using Shewhart control charts.

Results PDSAs of protocols preceded a large-scale rollout of a " 0.21 by 28 " campaign in 2014 leading to a special cause reduction in the "any BPD" rate, and a decrease in severe BPD (from 57 to 29\%). At the end of 2017, we reinvigorated the project, which led to dramatic decreases in the "any BPD" rate to $41 \%$ and the "severe BPD" rate to $21 \%$.

Conclusions A multidisciplinary QI initiative focused on process improvement geared towards the pathophysiological contributors of BPD has successfully reduced the rate of BPD in an all referral level IV NICU.
\end{abstract}

\section{Introduction}

\section{Available knowledge}

Bronchopulmonary Dysplasia (BPD), first described by Northway et al. [1], remains the most common complication of prematurity [2]. BPD infants have higher incidence of sepsis, intraventricular hemorrhage, mortality, readmission after discharge, and long-term neurodevelopmental delays [3]. BPD incidence is inversely correlated with gestational age at delivery and is the highest among infants born $<1000$ g (extremely low birthweight (ELBW)) [3]. Since the incidence of BPD is significantly higher among ELBW infants, transfer to tertiary centers for definitive care is

Supplementary information The online version of this article (https:// doi.org/10.1038/s41372-019-0574-8) contains supplementary material, which is available to authorized users.

Thomas Bartman

Thomas.Bartman@nationwidechildrens.org

1 Division of Neonatology, Nationwide Children's Hospital, Columbus, $\mathrm{OH}$, USA

2 Research Institute and Center for Perinatal Research, Nationwide Children's Hospital, Columbus, OH, USA

3 Nationwide Children's Hospital, Columbus, OH, USA required where, in some studies, the rate of BPD approaches $100 \%$ [4]. While advances have significantly improved ELBW survival, BPD rates have not improved [5].

Definitions of BPD vary, although the most accepted definition is the need for oxygen or respiratory support at 36-weeks postmenstrual age (PMA). Infants on room air at 36-weeks PMA do not have BPD. Infants requiring an effective fraction of inspired oxygen $\left(\mathrm{FiO}_{2}\right)<30 \%$ and not requiring positive pressure have mild $\mathrm{BPD}$, and those with an effective $\mathrm{FiO}_{2} \geq 30 \%$ and/or positive pressure have severe BPD [6, 7]. Severe BPD is further subclassified as type 1 (continuous positive airway pressure (CPAP) or high-flow nasal-cannula (HFNC; >21/min flow) at 36weeks PMA) and type 2 (ventilator dependence at 36weeks PMA). Infants with type 2 severe BPD more often have severe complications, including pulmonary hypertension, poor growth, and neurodevelopmental problems $[7,8]$.

Numerous medical management interventions have been proposed to prevent BPD, including avoiding mechanical ventilation, using "gentle ventilation" strategies (such as administrating CPAP in the delivery room and using bubble CPAP), and intubation/extubation for surfactant instillation. However, none of these in isolation have conclusively proven to prevent BPD. Several studies have demonstrated increases in BPD incidence even as BPD prevention strategies have been successfully implemented [9]. 


\section{Problem description}

We recognized that our referral level IV Neonatal Intensive Care Unit (NICU) had a relatively high incidence of BPD compared with similar units, which had worsened from 2012 to 2013. Therefore, in 2014, we inaugurated a project to reduce BPD incidence through quality improvement (QI) methodology.

Our specific AIM was to decrease the rate of any BPD from 73 to 58\% (a 20\% reduction of baseline) by August 31, 2018 for infants born under 32 0/7 weeks' gestation, admitted to the Nationwide Children's Hospital (NCH) main campus nursery by day-of-life (DOL) 28. Therefore, we excluded infants admitted at or above 36-weeks PMA as their diagnosis of BPD would be prior to arrival at our NICU.

We hypothesized that all practitioners might not be aware of our local problem, and were preoccupied with other QI efforts, suggesting that an intensive effort to educate and change culture would be necessary and sufficient to create improvement. We created protocols addressing oxygen management in the first month of life and invasive and noninvasive ventilation strategies, and instructed that all providers were responsible for all care (360-degree accountability).

\section{Context}

This initiative occurred at a tertiary, all referral children's hospital with 114 neonatal beds and $\sim 850$ neonatal admissions per year. $\mathrm{NCH}$ is a unique network of NICUs, with three physician practices (one academic and two private) admitting and caring for neonates at the tertiary care center ("main campus"). NCH also owns one Level II and five Level III NICUs regionally, operated by the same three physician groups who triage the sickest infants (usually infants with morbidities requiring other pediatric specialists) to the main campus. The infants were cared for in the main campus NCH Level IV NICU that includes the Comprehensive Center for Bronchopulmonary Dysplasia (a 24-bed unit dedicated to the care of the sickest BPD patients referred nationally [10]) and a small baby unit (a specially trained team, including physicians, nurses, physical and occupational therapists and nutritionists, caring for infants born at less than 27 weeks, as previously described [11]).

We created a multidisciplinary QI team (physicians, bedside nursing leaders, respiratory therapists (RT), administrative leadership, nurse practitioners, data analysts, and clinical therapists) with the mandate to decrease BPD in the high-risk population of the main campus NICU. Clinical practice guidelines [12] for the prevention and management of BPD were developed to optimize several lung-protective strategies based on the current available systematic review of the evidence. Because of NCH's focus on quality and safety $[13,14]$, our team included multiple individuals with formal training in the Institute for Healthcare Improvement (IHI) methodology [15], and the project had senior leadership support.

\section{Interventions}

The target and measured population for this project were infants born under $320 / 7$ weeks' gestation, admitted to the $\mathrm{NCH}$ main campus nursery by DOL 28 . We used IHI methodology and created our key driver diagram (Supplementary Fig. 1).

In response to our high rates of BPD and severe BPD, in 2014, we focused on two primary drivers: Oxygen management in the first month and ventilation strategies. We initiated three primary interventions via several plan-dostudy-act (PDSA) cycles. Because the interventions focused on developing protocols, all PDSAs involved having progressively larger numbers of individuals from multiple disciplines test the practicality of the protocols and provide feedback. Our protocol development targeted three major pathophysiological contributors to BPD. First, to reduce pulmonary barotrauma, we developed an RT-driven protocol to speed extubation of infants in the NICU (Supplementary Fig. 2). Second, to reduce oxygen toxicity, we revised our oxygen saturation guidelines and emphasized the goal of being on $21 \%$ oxygen by DOL 28 (" 0.21 by 28 " campaign). The target saturations for all infants under 32 weeks PMA, which had previously varied by provider, was standardized at $90-95 \%$. Third, to reduce oxygen need, scarring, and inflammation from atelectasis, we developed a noninvasive respiratory support protocol encouraging the use of CPAP through 36-weeks PMA unless the infant could come off CPAP without requiring more than minimal oxygen beyond 32 weeks PMA (Supplementary Fig. 3). The intention of these efforts were to minimize practice variations in respiratory modalities offered to the babies. The three protocols were introduced simultaneously in April 2014.

Following these interventions, we saw improvement in oxygen use at DOL 28 and severe BPD, but the rates returned to initial baseline or worse in 2016. We suspected that this was multifactorial: (1) incomplete buy-in from some staff into the guidelines, (2) ineffectiveness of education as the main intervention, (3) continual staff turnover, and (4) inability to keep focus on BPD prevention with multiple other QI initiatives taking place. For example, internal data collected through 2017 showed poor compliance with saturation alarm limit settings, with many set inappropriately at $100 \%$. Therefore, in late 2017 we launched a "reinvigoration" of the project and added more interventions based on the two primary drivers. We 
appointed a lead RT person during the days whose primary focus was to monitor compliance to protocols and saturation limits that were set on the monitor. In efforts to objectively and consistently manage supplemental oxygen in real time, we initiated the practice of utilization of $\mathrm{SpO} 2$ histograms.

Finally, we created weekly rounds (called "Cure Me" rounds) to improve process adherence to the interventions from 2014. The "Cure Me" rounds, led by a nurse and RT project-champion included education on the physiology of neonatal lung development, oxygen management guidelines, appropriate saturation alarm limits, target saturations, use of oxygen saturation histograms to evaluate oxygenation needs, and proper use of the standardized protocol for noninvasive positive pressure support. Protocol deviations were fixed during rounds and providers given real-time feedback about their practice variation.

To create a more effective sustain, an RT now randomly audits saturation alarm limits and histograms on a weekly basis to monitor compliance and provide real-time feedback and reinforcement to the "Cure Me" rounds. We review these results at our monthly meetings.

\section{Measures}

The primary outcome measures were the percentage of infants developing "any BPD" (presence of any oxygen and/or any respiratory modality at 36-weeks PMA) and "severe BPD" $\left(\mathrm{FiO}_{2}>30 \%\right.$ and/or positive pressure ventilation and/or HFNC at 36-weeks PMA) developed according to the definitions produced by the Children's Hospital Neonatal Database [16]. Process measures included percent of babies on oxygen on DOL 28, time to first extubation attempt, the ratio of CPAP to endotracheal tube (ETT) days and compliance with setting appropriate saturation alarm limits. The balancing measure was skin pressure injuries related to CPAP. Infants who did not survive to 36-weeks PMA were excluded from analysis. All data were obtained from our internal abstracted database or from random manual audits (saturation alarm limits).

\section{Analysis and ethical considerations}

Data were plotted on control charts using statistical process control charts, as well as a Microsoft Excel plug-in coded by this institution's statistician. We used standard Nelson Rules and rules from the American Society for Quality to determine statistical significance. All centerlines are means. Upper and lower control limits (expected bounds of performance, giving the limits of common cause variation) are shown on the charts. Specific rules allowing a shift included 8 points in a row, or 10 points out of 11 , or 12 points out of 14 above or below a centerline (http://asq.org/learn-aboutquality/data-collection-analysis-tools/overview/control-cha rt.html). Other rules for special cause were used once [17]. This project was classified by the institutional review board as QI work and did not require full review.

\section{Results}

The average gestational age at birth in this cohort was 27.7 weeks CGA in 2012, declined to 26.9 in early 2013, and then increased to 27.6 in late 2014 and has remained constant. Birthweight throughout the project averaged 1081 g (SD $424 \mathrm{~g}$ ) with no trends. DOL at transfer to the main campus NICU averaged 7.8 days (SD 8.2 days) with no trends. Mortality rates in our dataset over this period did not change significantly (data not shown). When we started the project in January of 2014 (after all three tracked metrics had control chart shifts in an undesired direction in 2013) our baseline rates were $80 \%$ (of supplemental oxygen at DOL 28), $73 \%$ (outcome measure of infants developing any BPD), and $57 \%$ (of infants developing severe BPD) (Figs. 1-3).

Following PDSAs culminating in a large-scale "rollout" of the " 0.21 by 28 " campaign in April 2014, the centerlines shifted in oxygen use at DOL 28 and severe BPD. The percent of infants on oxygen at DOL 28 decreased to $53 \%$ and the percent of infants with severe BPD decreased to 29\%, with all shifts occurring between April and August 2014 (Figs. 1 and 3). The rate of any BPD did not show a shift, but a 4-month-long special cause signal was seen shortly after the interventions (Fig. 2). Another protracted period of statistically significant improvement in the any BPD rate was seen for 13 months in 2015-2016, although not meeting "traditional" SPC rules [17].

In 2016, the two metrics which had shown improvement worsened. At the end of 2017, we selected a new goal to decrease any BPD to $40 \%$, reinvigorated the project, and introduced "Cure Me" rounds, which led to improvement in all three metrics to the lowest levels seen over the 6 years tracked on the control charts (Figs. 1-3).

The ratio of the number of days on CPAP to the number of days with the invasive ETT increased gradually from 2015 to 2017 and dramatically in 2018 (Fig. 4). While this metric cannot be temporally linked to outcomes (due to annualized values), the data suggest improvement in reduction of ETT days and increase in CPAP days, both of which were desired results from the interventions. Compliance with setting appropriate saturation alarm limits is $\sim 90 \%$, and the time to first extubation attempt decreased from 34 days to 10 days in May 2015 and has been sustained (Supplementary Fig. 4).

We further evaluated the subset of severe BPD patients, on mechanical ventilation. The proportion of infants on invasive mechanical ventilation at 36-weeks PMA (Type 


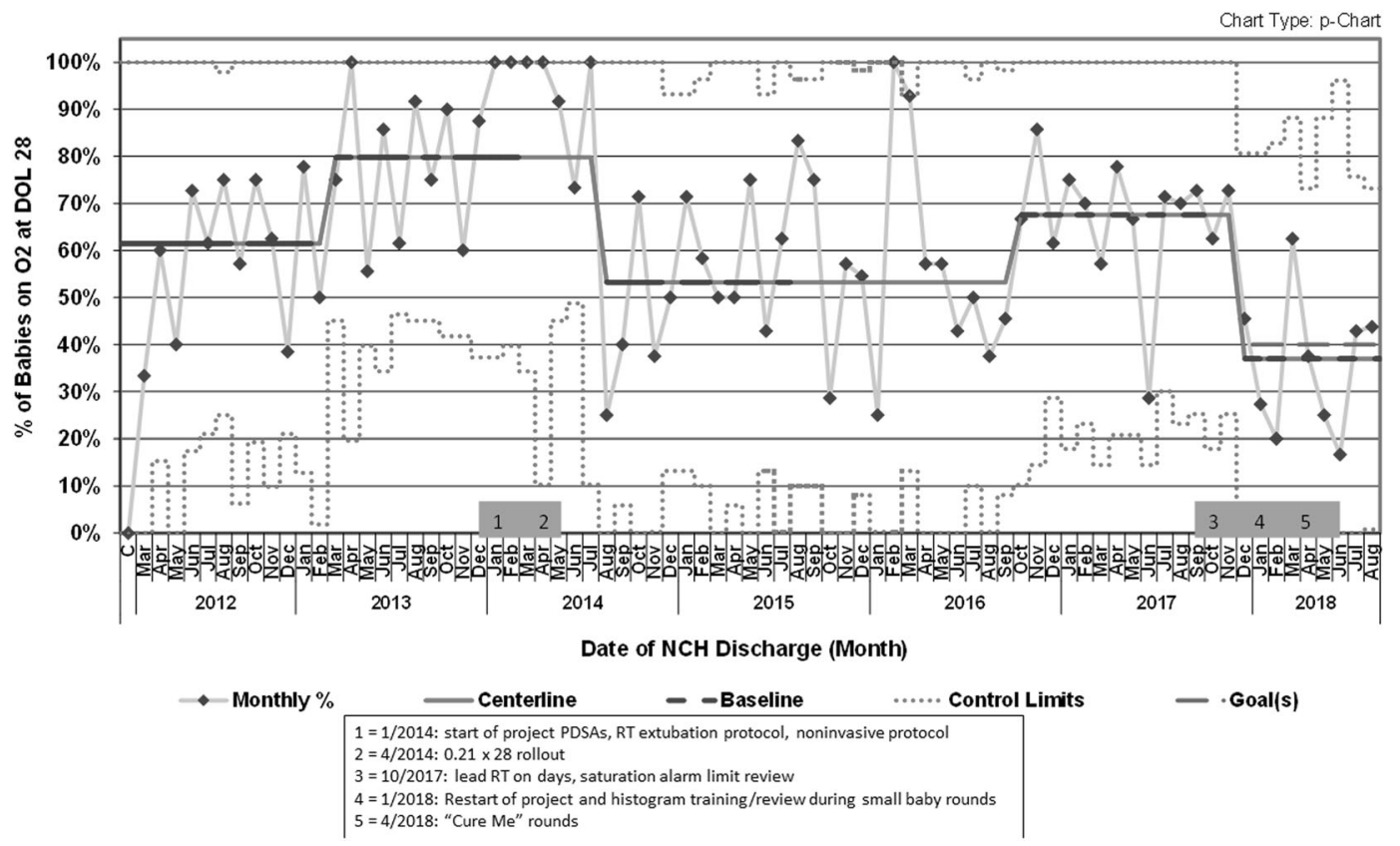

Fig. 1 p control chart showing percent of babies in cohort population requiring oxygen on day-of-life 28 . Solid red $=$ centerline (mean). Dashed blue $=$ points used for baseline calculation. Dashed red $=$ control limits. Dashed green $=$ goal. Shifts meet ASQ rules (March $2013=12$ out of 14 as July 2013 is above previous centerline;

2 severe BPD as explained above) fell from $47 \%$ in 2014 to 4\% in 2018 (data not shown).

Because our initiative was expected to create a much higher use of CPAP, and respiratory devices are a common cause of pressure injuries, our balancing measure was skin pressure injuries related to CPAP. Fortunately, our NICU had a QI initiative to decrease pressure ulcers, which coincided with this project [18]. During these projects, pressure ulcers decreased from 0.6 per 100 CPAP days in 2014 to 0.27 per 100 CPAP days in 2018 .

\section{Discussion}

We describe our experience with a multidisciplinary QI effort to decrease the incidence of BPD in an all referral Children's Hospital NICU. Despite decades of BPD studies, many recent cohorts of ELBW infants have shown an increase in the BPD incidence $[5,19]$. Thus, strategies to decrease the incidence of BPD have been of high priority to neonatologists [20]. The key drivers in our QI project were derived from the known physiologic mechanisms leading to BPD [21]. Our interventions included those that have shown evidence of reducing the incidence of BPD [20, 22]. The combination of guideline development/rollout was only partially and temporarily effective, until consistent project champions could provide timely, non-punitive actionable feedback to the front line staff to improve adherence ("Cure Me" rounds). This observation
August $2014=8$ in a row; October $2016=8$ in a row; December $2017=8$ in a row). 12 points were used to calculate all centerlines. Centerlines starting in 2013 are $80 \%, 53 \%, 68 \%$, and 37\%. (Color figure online).

underscores the need to monitor improvements during a sustain phase, in case a new system has not become fully embedded into the culture [23].

Our " 0.21 by 28 " efforts demonstrate that a conscious effort to reduce $\mathrm{FiO}_{2}$ while maintaining $\mathrm{SpO}_{2}$ goals in the first 28 days-of-life resulted in improved pulmonary outcomes at 36-weeks PMA. Our results are consistent with a recent study by Wai et al. [22], wherein a secondary analysis of the Trial of Late SURFactant to Prevent BPD data demonstrated that a lower cumulative supplemental oxygen exposure at DOL 14 correlated with a lower incidence of BPD or death [24, 25]. Similarly, Stevens et al. [26] reported that the area under the supplemental oxygen curve at DOL 72-h was a predictor of later respiratory symptoms and need for medications to treat them in very low birthweight infants.

The need for mechanical ventilation has been linked to the development of BPD, and despite no randomized controlled trials, avoidance of invasive mechanical ventilation correlates with lower rates of BPD in preterm infants $[27,28]$. Therefore, another part of our multidisciplinary approach to BPD reduction was to develop and implement a respiratory-therapist-driven extubation-protocol for this population. In this high-risk population of preterm infants, we were able to extubate babies sooner and use more noninvasive positive pressure support, which correlated with a decrease in our rates of any BPD and severe BPD over the same period. These results are consistent with our previous 


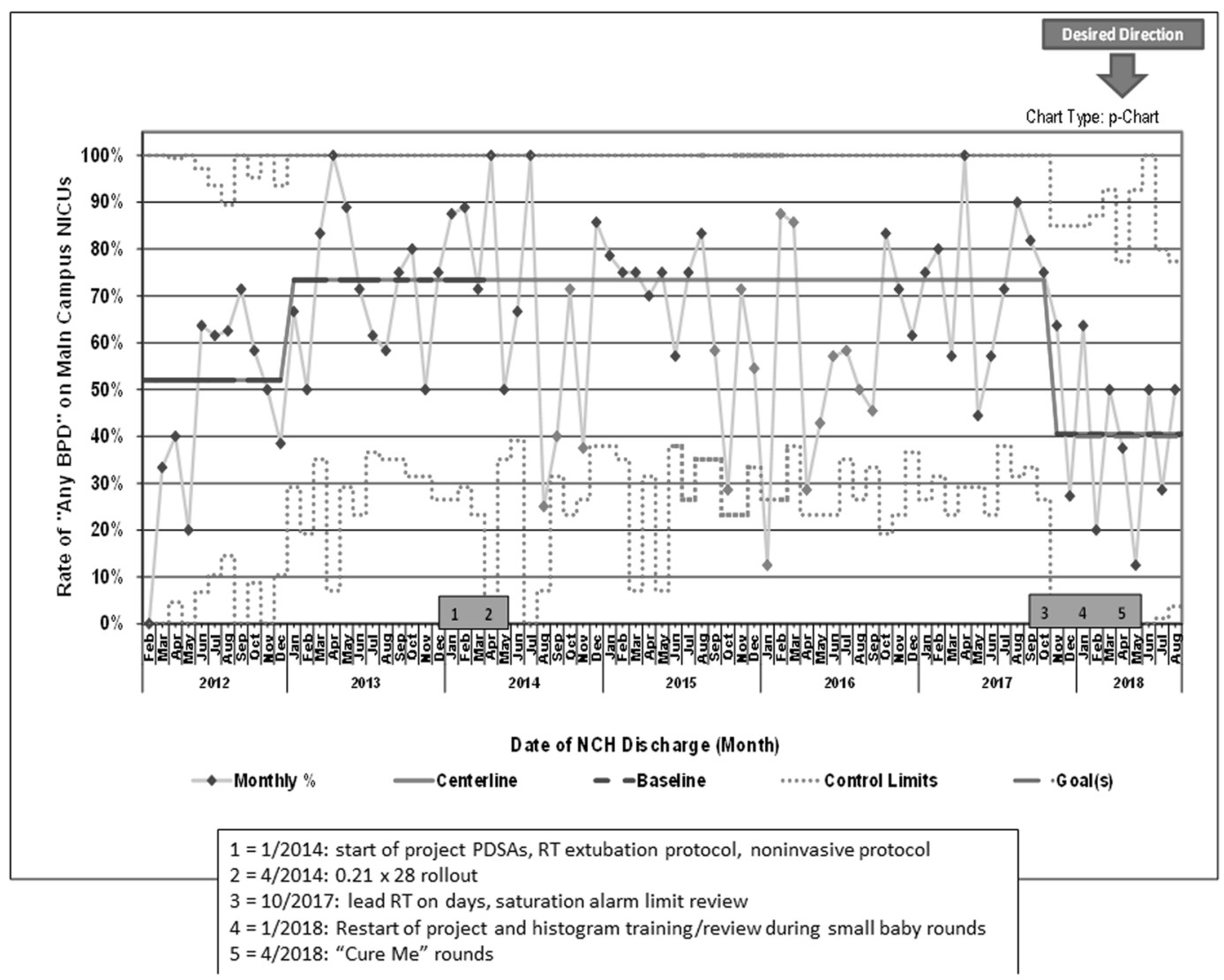

Fig. 2 p control chart showing percent of babies with any BPD at 36 weeks corrected gestational age. Solid red = centerline (mean). Dashed blue $=$ points used for baseline calculation. Dashed red $=$ control limits. Dashed green $=$ goal. Shifts meet ASQ rules (January $2013=12$ out of 14 ; November $2017=8$ in a row). 12 points were used to calculate all centerlines. Two other special cause signals are

data from our extremely low gestational age patients, which demonstrated that the earlier an extubation attempt was made, the lower the rate of BPD or severe BPD, even if reintubation was required [29]. During the process of this project, since we anticipated increase in rates of CPAP, we undertook proactive measures to minimize skin pressure injuries, such as weekly dedicated skin rounding team, daily evaluation and vigilance by both RT and bedside nurse.

If we consider only those infants with severe BPD, the proportion of infants on invasive mechanical ventilation at 36-weeks PMA fell from 47\% in 2014 to $4 \%$ in 2018 (data not shown), indicating that this QI effort reduced the worst of the worst outcomes. Abman et al. [8] suggested that infants with severe BPD comprise a very heterogeneous group with a wide range of "severity" and that one could begin to assess the severity using the need for invasive mechanical ventilation as a marker. Our findings suggest that even when the infant develops severe BPD, efforts to extubate patients earlier can attenuate the severity of the disease. indicated by red points: August-November $2014=2$ out of 3 in outer third of control limit; September 2015-September 2016 = run which meets rule for statistical significance without meeting "traditional" SPC rules (see Wheeler et al. [17]). Centerlines are 52\%, 73\%, and $41 \%$. (Color figure online).

In this QI initiative, we have thus far not evaluated the direct effect of our interventions on subsets of gestational age, associated neonatal morbidities, such as length of stay, tracheostomy rates, retinopathy of prematurity, or neurodevelopmental outcomes. We hope to evaluate these in the future. Our team also hopes to focus on other drivers such as preadmission respiratory management, nutrition, and other comorbidities as mentioned in the key driver diagram which may have an impact on decreasing the rates of BPD. No standardized changes in use of steroids or other medications were introduced in our NICU during this project. As our next step, we look forward to utilizing these standardized measures, real-time data compliance, and multidisciplinary collaboration with other centers to improve care for this population.

In summary, we describe a multidisciplinary QI initiative to decrease our rates of any BPD and severe BPD. The QI project utilized key drivers with design changes/interventions based on physiologic pulmonary mechanics and focused on process improvement. Using this approach, we 


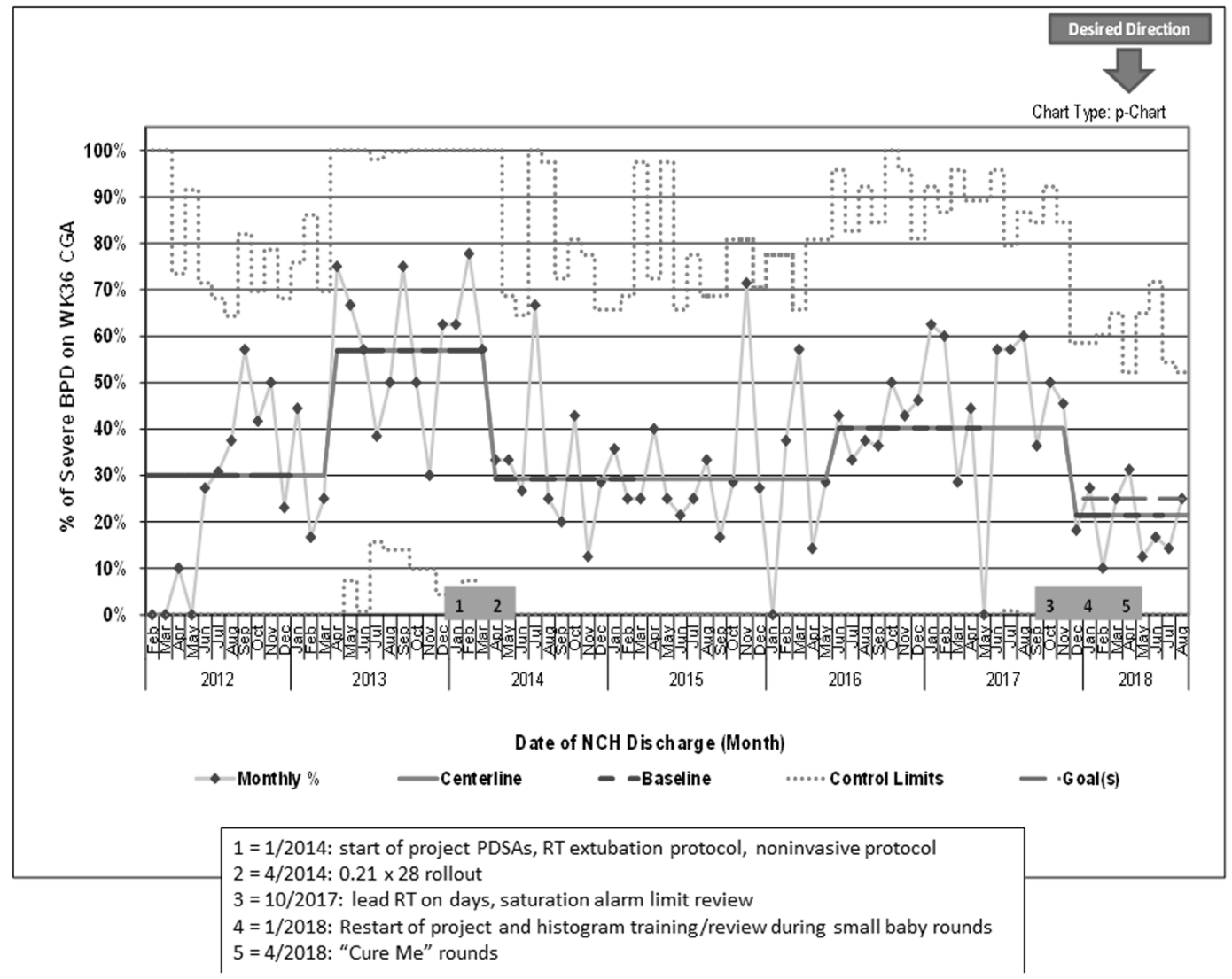

Fig. 3 p control chart showing percent of babies in cohort population with severe BPD at 36 weeks corrected gestational age. Solid red $=$ centerline $($ mean). Dashed blue $=$ points used for baseline calculation. Dashed red $=$ control limits. Dashed green $=$ goal. Shifts

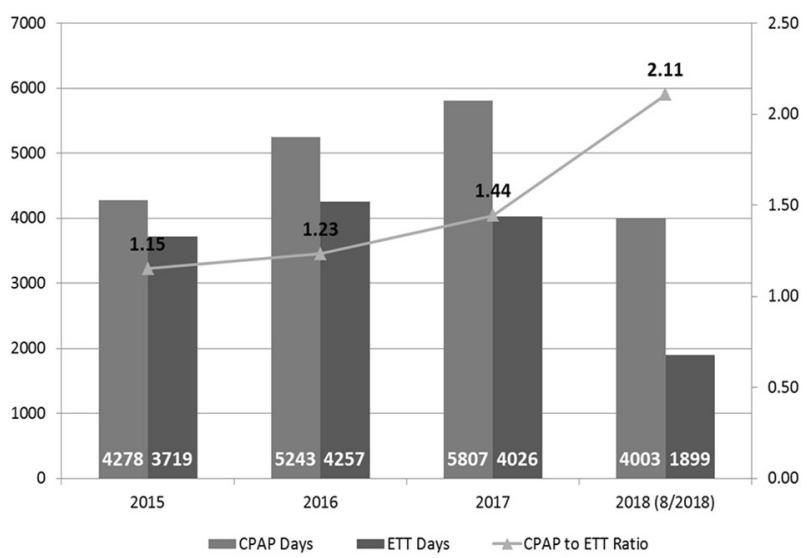

Fig. 4 Number of CPAP days (blue bars), ETT days (red bars) and ratio of CPAP to ETT days (green line), by year. 2018 is through the end of August, 2018. (Color figure online).

demonstrate a reduction in the rate of BPD in the NICU of our all referral Children's Hospital Level IV NICU from $73 \%$ in 2013 to $41 \%$ in 2018 and in severe BPD from $57 \%$ in 2013 to $21 \%$ in 2018 . The number of infants admitted or the severity of their illness during that period were not meet ASQ rules (April $2013=10$ out of 11; April 2014 $=10$ out of 11; June $2016=8$ in a row; December $2017=8$ in a row). 12 points were used to calculate all centerlines. Centerlines starting in mid-2013 are $57 \%, 29 \%, 40 \%$, and $21 \%$. (Color figure online).

substantially changed. Our data suggest that a focused, multidisciplinary, stringent QI approach to BPD reduction focused on process improvement can substantially improve BPD rates.

\section{Conclusions}

The definition, pathophysiology, and management of BPD has evolved significantly since first described by Northway almost 50 years ago. Advances in neonatal care have resulted in increased rates of survival of extremely premature infants leading to an emerging population of longterm survivors of BPD. In this paper, we highlight our interdisciplinary QI efforts to address the complex physiologic pulmonary mechanisms of these patients that are critical and influence outcomes of BPD.

Author contribution RB, ES, LN, GR, and TB conceptualized and designed the study, coordinated and supervised data collection, drafted the initial manuscript, and reviewed and revised the manuscript. AE designed the data collection instruments, collected data, carried out the 
initial analyses, and reviewed and revised the manuscript. All authors approved the final manuscript as submitted and agree to be accountable for all aspects of the work.

\section{Compliance with ethical standards}

Conflict of interest The authors declare that they have no conflict of interest.

Publisher's note Springer Nature remains neutral with regard to jurisdictional claims in published maps and institutional affiliations.

\section{References}

1. Northway WH Jr., Rosan RC, Porter DY. Pulmonary disease following respirator therapy of hyaline-membrane disease. Bronchopulmonary dysplasia. N. Engl J Med. 1967;276:357-68.

2. Doyle LW, Anderson PJ. Long-term outcomes of bronchopulmonary dysplasia. Semin Fetal Neonatal Med. 2009;14:391-5.

3. Ehrenkranz RA, Walsh MC, Vohr BR, Jobe AH, Wright LL, Fanaroff AA, et al. Validation of the National Institutes of Health consensus definition of bronchopulmonary dysplasia. Pediatrics. 2005;116:1353-60.

4. Walsh MC, Szefler S, Davis J, Allen M, Van Marter L, Abman S, et al. Summary proceedings from the bronchopulmonary dysplasia group. Pediatrics. 2006;117:S52-6.

5. Stoll BJ, Hansen NI, Bell EF, Walsh MC, Carlo WA, Shankaran $\mathrm{S}$, et al. Trends in care practices, morbidity, and mortality of extremely preterm neonates, 1993-2012. J Am Med Assoc. 2015;314:1039-51.

6. Jobe AH, Bancalari E. Bronchopulmonary dysplasia. Am J Respir Crit Care Med. 2001;163:1723-9.

7. Higgins RD, Jobe AH, Koso-Thomas M, Bancalari E, Viscardi RM, Hartert TV, et al. Bronchopulmonary dysplasia: executive summary of a workshop. J Pediatr. 2018;197:300-8.

8. Abman SH, Collaco JM, Shepherd EG, Keszler M, Cuevas-Guaman M, Welty SE, et al. Interdisciplinary care of children with severe bronchopulmonary dysplasia. J Pediatr. 2017;181:12-28 e1.

9. Zysman-Colman Z, Tremblay GM, Bandeali S, Landry JS. Bronchopulmonary dysplasia-trends over three decades. Paediatr Child Health. 2013;18:86-90.

10. Shepherd EG, Knupp AM, Welty SE, Susey KM, Gardner WP, Gest AL. An interdisciplinary bronchopulmonary dysplasia program is associated with improved neurodevelopmental outcomes and fewer rehospitalizations. J Perinatol. 2012;32:33-8.

11. Nankervis CA, Martin EM, Crane ML, Samson KS, Welty SE, Nelin LD. Implementation of a multidisciplinary guideline-driven approach to the care of the extremely premature infant improved hospital outcomes. Acta Paediatr. 2010;99:188-93.

12. Institute of Medicine (US) Committee on Standards for Developing Trustworthy Clinical Practice Guidelines; Graham R, Mancher M, Miller Wolman D, et al., editors. Clinical Practice Guidelines We Can Trust. Washington (DC): National Academies Press (US); 2011. 6, Promoting Adoption of Clinical Practice Guidelines. Available from: https://www.ncbi.nlm.nih.gov/books/ NBK209543/.
13. Brilli RJ, Allen S, Davis JT. Revisiting the quality chasm. Pediatrics. 2014;133:763-5.

14. Brilli RJ, Crandall WV, Berry JC, Stoverock L, Rosen K, Budin L, et al. A patient/family-centered strategic plan can drive significant improvement. Adv Pediatr. 2014;61:197-214.

15. Langley GJ. The improvement guide: a practical approach to enhancing organizational performance. 2nd ed. San Francisco: Jossey-Bass; 2009.

16. Murthy K, Porta NFM, Lagatta JM, Zaniletti I, Truog WE, Grover TR, et al. Inter-center variation in death or tracheostomy placement in infants with severe bronchopulmonary dysplasia. J Perinatol. 2017;37:723-7.

17. Wheeler TA, Davis JT, Brilli RJ. The aggregate point rule for identifying shifts on $\mathrm{P}$ charts and $\mathrm{U}$ charts. Pediatr Qual Saf. 2018;3:e103.

18. Nist MD, Rodgers EA, Ruth BM, Bertoni CB, Bartman T, Keller LA, et al. Skin rounds: a quality improvement approach to enhance skin care in the neonatal intensive care unit. Adv Neonatal Care. 2016;16 Suppl 5S :S33-41.

19. Doyle LW, Carse E, Adams AM, Ranganathan S, Opie G, Cheong JLY, et al. Ventilation in extremely preterm infants and respiratory function at 8 years. N Engl J Med. 2017;377:329-37.

20. Nelin LD, Bhandari V. How to decrease bronchopulmonary dysplasia in your neonatal intensive care unit today and "tomorrow". F1000Res. 2017;6:539.

21. Jobe AH. The new BPD. NeoReviews. 2006;7:e531-45.

22. Wai KC, Kohn MA, Ballard RA, Truog WE, Black DM, Asselin JM, et al. Early cumulative supplemental oxygen predicts bronchopulmonary dysplasia in high risk extremely low gestational age newborns. J Pediatr 2016;177:97-102 e2.

23. Lennox L, Maher L, Reed J. Navigating the sustainability landscape: a systematic review of sustainability approaches in healthcare. Implement Sci. 2018;13:27.

24. Keller RL, Eichenwald EC, Hibbs AM, Rogers EE, Wai KC, Black DM, et al. The randomized, controlled trial of late surfactant: effects on respiratory outcomes at 1-year corrected age. J Pediatr. 2017;183:19-25 e2.

25. Ballard RA, Keller RL, Black DM, Ballard PL, Merrill JD, Eichenwald EC, et al. Randomized trial of late surfactant treatment in ventilated preterm infants receiving inhaled nitric oxide. J Pediatr. 2016;168:23-9 e4.

26. Stevens TP, Dylag A, Panthagani I, Pryhuber G, Halterman J. Effect of cumulative oxygen exposure on respiratory symptoms during infancy among VLBW infants without bronchopulmonary dysplasia. Pediatr Pulmonol. 2010;45:371-9.

27. Subramaniam P, Ho JJ, Davis PG. Prophylactic nasal continuous positive airway pressure for preventing morbidity and mortality in very preterm infants. Cochrane Database Syst Rev. 2016:CD001243. https://doi.org/10.1002/14651858.CD001243. pub3.

28. Fischer HS, Buhrer C. Avoiding endotracheal ventilation to prevent bronchopulmonary dysplasia: a meta-analysis. Pediatrics. 2013;132:e1351-60.

29. Robbins M, Trittmann J, Martin E, Reber KM, Nelin L, Shepherd E. Early extubation attempts reduce length of stay in extremely preterm infants even if re-intubation is necessary. J Neonatal Perinat Med. 2015;8:91-7. 